\title{
PERCEPCIÓN, CONTENIDO INTENCIONAL Y SINGULARIDAD
}

\author{
Francisco Pereira \\ Universidad Alberto Hurtado \\ francisco@correo.org.uk
}

\begin{abstract}
Resumen
Muchos filósofos piensan que los contenidos perceptuales son siempre generales y que no deben especificarse utilizando términos singulares. Ellos creen que los objetos/propiedades distales no constituyen esencialmente los contenidos perceptuales. Argumentaré que esta estrategia es insatisfactoria al especificar contenidos e incapaz de dar cuenta de una intuición común, según la cual la veracidad de nuestras experiencias perceptuales no es algo evaluable con independencia de si percibimos o no. Finalmente, sugeriré una alternativa "singularista" en la que los objetos distales sí entran en los contenidos, haciendo de las percepciones y las alucinaciones dos tipos de experiencia fundamentalmente diferentes.
\end{abstract}

Palabras Clave: intencionalidad, contenido intencional, percepción visual, veracidad, generalismo, singularidad, conceptos demostrativos, Evans, McDowell, Sainsbury, Soteriou.

\section{Abstract}

Many philosophers think that perceptual contents are always general and shouldn't be specified using singular terms. They believe that distal objects and properties don't essentially constitute perceptual contents. I will argue that this strategy doesn 't provide a satisfactory account of content specification and fails to make sense of the common-sense intuition that the veridicality of our perceptual experiences isn't something fully independent of whether we perceive or not. Finally, I will suggest an alternative "singularist" standpoint in which distal objects do enter into visual contents, making perceptions and hallucinations fundamentally different types of experiences.

KEYWORDS: intentionality, intentional content, visual perception, veridicality, generalism, singularity, demonstrative concepts, Evans, McDowell, Sainsbury, Soteriou. 
$\overline{\mathrm{RA}}$ Una de las visiones filosóficas más difundidas dentro del debate contemporáneo acerca de la naturaleza de la percepción visual adopta una postura de corte intencional e internista. "Intencional", porque explica nuestra capacidad de ver cosas (objetos, propiedades) localizadas en nuestro entorno en términos de nuestra capacidad para tener experiencias visuales con contenidos intencionales, es decir, con contenidos que representan el mundo como siendo de tal y tal forma. "Internista", porque supuestamente aquellas cosas localizadas en nuestro entorno físico (objetos distales) no constituyen esencialmente los contenidos intencionales de nuestra experiencia. De hecho, según la interpretación descrita, la experiencia con contenido intencional de un sujeto $S$ que ve un objeto $O$, es en principio una experiencia del mismo tipo que la experiencia que $S$ podría tener si se encontrara meramente alucinando $O$.

Para ilustrar adecuadamente esta interpretación y ver en qué sentido se propone que los objetos distales no son componentes esenciales de los contenidos intencionales de la percepción, es fundamental que recordemos lo sugerido por Grice $(1961,1988)$ al plantear la posibilidad de un fenómeno que hoy conocemos como "alucinación verídica". El ejemplo de Grice nos permitirá introducir el supuesto carácter general de todos los contenidos de nuestra experiencia perceptual, asunto que será el tema central de discusión en este artículo. Imaginen que un neurocientífico estimula la corteza visual de un sujeto para inducir una experiencia en la cual para el sujeto es como si hubiera un reloj en la repisa frente a él.

Si aquella medida se aplicara cuando realmente había un reloj en la repisa, y si las impresiones [del sujeto] continuaran sin cambio alguno cuando el reloj se quitó o se alteró su posición, entonces yo pienso que debemos inclinarnos a decir que [el sujeto] no vio el reloj que estaba frente a sus ojos (Grice 1988, 69).

En este caso hipotético, el sujeto no ve el reloj, es decir, no percibe un objeto, a pesar de que en su entorno, frente a él, hay un objeto que es realmente tal como su experiencia lo representa. A juicio de Grice, la falla radica en que la experiencia no es causalmente dependiente de los objetos relevantes -el reloj, en este caso- de una manera apropiada. Desde una postura intencionalista compatible con la que hemos estado describiendo, la falla expuesta por Grice se explicaría mediante el siguiente análisis:

(a) S tiene una experiencia visual con un contenido intencional que se puede expresar en una sentencia de la forma "Me parece como si hubiera un reloj al frente mío".

(b) Hay un reloj en el entorno frente a $\mathrm{S}$.

(c) La experiencia visual de S no es causalmente dependiente (de la manera adecuada) del reloj.

(d) S no ve el reloj. 
Es importante recalcar que el elemento "internista" de la teoría intencional de la cual intentamos dar cuenta surge siempre y cuando aceptemos que la experiencia visual con contenido intencional presente en (a), es una experiencia fundamentalmente del mismo tipo que la experiencia que $\mathrm{S}$ tendría si estuviera efectivamente viendo o percibiendo el objeto en cuestión. Es decir, se trata de un tipo idéntico de experiencia consciente más allá (independientemente) de si S ve o alucina un reloj sobre la estantería. Si en todos los casos (percepción, ilusión o alucinación del reloj) se trata de una experiencia del mismo tipo, entonces el éxito logrado cuando de hecho percibimos algo, solo se explica por la presencia de lo que podríamos llamar una experiencia intencional común más la satisfacción de ciertas consideraciones extras de tipo causal y existencial.

Es precisamente este rol "extra" que juegan los objetos distales y las propiedades que percibimos lo que permite ilustrar la naturaleza internista de la teoría expuesta. Si un objeto percibido es meramente algo "extra", es decir, algo que no constituye de manera esencial el contenido intencional de nuestra experiencia visual, podría argumentarse que lo representado conscientemente durante la experiencia es claramente interno (en cuanto no está esencialmente constituido por el mundo externo). Esta supuesta independencia interna de los contenidos respecto de los objetos físicos que ellos representan ha tenido una fuerte impacto en la formulación o análisis clásico del concepto de percepción. De hecho, para muchos filósofos de orientación analítica, cualquier instancia de percepción debe analizarse bajo el paradigma de la satisfacción de tres condiciones individualmente necesarias y conjuntamente suficientes: (i) el tener una experiencia con contenido intencional, (ii) la existencia real de los objetos y propiedades que figuran en el contenido intencional en el entorno físico del sujeto que percibe, y (iii) la existencia de un vínculo o relación causal adecuada entre estos objetos y la experiencia del sujeto.

Hay dos aspectos que me preocupan profundamente respecto de esta supuesta naturaleza interna de los contenidos perceptuales que acabo de exponer. Se trata de dos tesis que parecen ser consecuencia directa de esta caracterización. Primero, si los objetos mundanos que percibimos no constituyen nuestras experiencias visuales y sus contenidos intencionales, entonces no parece ser posible asegurar que nuestras experiencias perceptuales son esencialmente relacionales, lo que a mi juicio contradice nuestras intuiciones más profundas con respecto a la percepción. De hecho, algunos intencionalistas internistas ya han admitido abiertamente este primer aspecto.

La postura intencional también tiene un precio. Ella debe negar que la experiencia perceptual es una relación. Cuando uno efectivamente tiene éxito al percibir un objeto, por supuesto, uno se relaciona con él; pero esta relación no es esencial para que la experiencia perceptual sea del tipo fundamental que es. Entonces, en cierta forma, los críticos del intencionalismo tienen la razón cuando dicen que la teoría intencional de la percepción "queda corta" de mundo... (Crane 2006, 141)

Si las experiencias que tenemos cuando vemos o alucinamos un objeto determinado son del mismo tipo o de la misma categoría ontológica, no es posible afirmar 
que los objetos "entran" o constituyen esencialmente los contenidos de estas experiencias. Literalmente, las experiencias y sus contenidos son algo "extra" y quedan "cortas de mundo". Esta es una de las razones por la que muchos teóricos contemporáneos aceptan sin mayor cuestionamiento la verdad de una segunda tesis. Me refiero a la tesis de los contenidos "generales". Si aceptamos la sugerencia de Crane y otros internistas cuando dicen que los objetos mundanos no constituyen esencialmente los contenidos intencionales en los que figuran, tendríamos también que aceptar que todos los contenidos de la percepción son siempre "generales", es decir, que ellos nunca involucran esencialmente objetos particulares y, por lo mismo, que no deben especificarse usando términos singulares.

En lo que sigue centraré mis reflexiones en la tesis de los contenidos generales. Argumentaré que el generalismo presenta serias dificultades y que no provee una visión satisfactoria de la naturaleza de los contenidos de la percepción. Las dificultades del generalismo nos llevarán a buscar una alternativa que llamaré "singularista", según la cual los objetos sí juegan un rol esencial en la constitución de los contenidos intencionales de la percepción. Si esto es así, entonces las relaciones con los objetos del mundo sí son cruciales y juegan un rol constitutivo fundamental. Desde una perspectiva metodológica, mi artículo intentará reposicionar el aspecto esencialmente relacional de la percepción visual ${ }^{1}$.

\section{Generalismo y percepción verídica incorrecta}

Existe una teoría muy difundida en la filosofía de la mente -llamémosla "generalismo"- que dice que los contenidos de la experiencia perceptual son siempre generales, es decir, no incluyen esencialmente a los objetos de la percepción y no deberían especificarse utilizando términos singulares. Por ejemplo, según Colin McGinn, "los ítems en el mundo que poseen todas las propiedades especificadas por el contenido de la experiencia 'calzan' o se 'adecuan' al contenido de la experiencia” $(1982,51)$. De un modo similar, Michael Tye ha dicho recientemente que el contenido intencional de una percepción debe ser abstracto, esto es, debe ser un "contenido en el que ningún objeto concreto particular o superficie entra" $(2000,62)$.

Como vimos en la introducción de este artículo, una de las razones principales para justificar el generalismo es la posibilidad de tener una experiencia visual con un contenido que "calza" o se "adecua" al mundo, pero que carece de los vínculos causales necesarios para ser estrictamente una percepción. Dado que la ausencia de conexiones causales adecuadas no impide que los contenidos de estas experiencias sean correctos,

1 Utilizaré los términos "experiencia perceptual", "percepción" y "contenido perceptual" en relación exclusiva con aquellos casos en que efectivamente un sujeto está percibiendo el entorno. Utilizaré las expresiones "experiencia visual" o "contenido visual" de manera neutral para referirme indistintamente, ya sea a experiencias perceptuales, ilusorias o alucinatorias. 
el generalismo no es solo una propuesta implícitamente conectada con la idea de que el contenido de la percepción es siempre general y existencialmente cuantificado, sino también con la idea de que las alucinaciones verídicas son posibles.

No se requiere mucho esfuerzo para percatarse de que el generalismo está en tensión con la idea de que los objetos distales (los objetos localizados en nuestro entorno) determinan constitutivamente el tipo de experiencia que tenemos. El generalismo parece ir de la mano con la posibilidad de tener esencialmente un mismo tipo de experiencia intencional, independientemente de si percibimos o no. Esto contrasta con la lectura de re, según la cual percibir es necesariamente relacionarse con el entorno de una manera fundamental, es decir, relacionarse de manera que los contenidos de nuestra experiencia (y por tanto el tipo de experiencia que tenemos) dependan de la existencia de objetos particulares en el mundo.

A continuación, sobre la base de algunas reflexiones de Sainsbury (2005) y Soteriou (2000), argumentaré que el generalismo no provee una teoría satisfactoria de los contenidos perceptuales. Frente a los diversos problemas que a mi juicio presenta el generalismo, sugeriré que es necesario buscar una alternativa singularista, es decir, una alternativa en que los objetos particulares del entorno sí entran en los contenidos perceptuales y en la que la supuesta "veracidad" de nuestra experiencia (si nuestras experiencias con contenido intencional representan o no adecuadamente nuestro entorno) es algo que no puede decidirse independientemente de si hay o no hay objetos / propiedades relacionándose con nuestra experiencia en un momento determinado ${ }^{2}$.

¿Por qué debemos aceptar la intuición singularista? ¿Qué tiene de malo aceptar que la veracidad de una experiencia es algo independiente de si dicha experiencia es o no una percepción, tal como lo sugieren las alucinaciones verídicas de Grice (1988)? Para comenzar a dar una respuesta coherente a este problema -asumiendo que el contenido de una experiencia visual puede al menos ser descrito ${ }^{3}$ en forma proposicional- focalicemos nuestra discusión en cómo los objetos se presentan o se muestran durante la percepción. Para ello podemos utilizar expresiones del llamado lenguaje de las apariencias, tales como "Este lápiz parece verde" o "Aquel vidrio parece estar trizado".

Los contenidos de las experiencias que reportamos utilizando el lenguaje de las apariencias son verídicos si y solo si los objetos y propiedades representadas son realmente tal como parecen ser desde la perspectiva del sujeto. Generalizando, podemos decir que "para cualquier objeto $x$ y cualquier forma en la que un objeto puede

2 He traducido el término inglés "veridicality" por "veracidad". Es importante recordar que utilizo esta expresión en el sentido técnico con que se utiliza en psicología y filosofía de la mente, es decir, para referirme exclusivamente al hecho de que una experiencia representa correctamente objetos y propiedades en el entorno. Aquí, como en muchos otros pasajes, agradezco los agudos comentarios y sugerencias del corrector anónimo de este artículo.

3 Digo que al menos pueden ser "descritos" como una forma de distinguir mis supuestos de la idea de que los contenidos perceptuales son totalmente proposicionales. 
parecer $F$, si $x$ es experimentado como $F$, la experiencia es verídica con respecto a $x$ y $F$ si y solo si $x$ es $F^{\prime \prime}$ (Sainsbury 2005, 243). Aparte del elemento predicativo presente en un contenido intencional, como es verde o está trizado, para que ese contenido sea evaluado como verdadero o falso, también debe poseer un elemento que denote el objeto supuestamente percibido por el sujeto. El contenido podría tener la forma "Existe un $x$ que es $F$ " o alternativamente "Ese $x$ es $F$ " 4 . Teniendo este esquema teórico a la vista, detengámonos un momento a evaluar la tesis generalista -la visión filosófica según la cual los contenidos de la percepción son generales- tal como es usualmente presentada en los debates contemporáneos. Adoptando la interpretación que nos ofrece Mark Sainsbury $(2005,47)$, el generalismo parece ser a menudo formulado de tres maneras diferentes. Cada una de estas formulaciones conlleva importantes problemas que justifican la búsqueda una alternativa singularista ${ }^{5}$ :

- [Existencialismo] Un contenido completo puede ser especificado utilizando una descripción indefinida sin condición singularizadora alguna.

- [Russellianismo Puro] Un contenido completo puede ser especificado utilizando una descripción definida que solo contiene términos generales.

- [Russellianismo Impuro] Un contenido completo puede ser especificado utilizando una descripción definida que contiene algunos términos singulares para una restringida gama de entidades específicas.

Es importante recalcar que hay problemas bastante familiares respecto al "existencialismo", específicamente en relación con lo que podríamos denominar el rastreo perceptual de objetos. Está claro que uno y el mismo objeto particular $O_{1}$, puede estar en una localización particular $l_{1}$, en un tiempo determinado $\mathrm{t}_{1} \mathrm{y}$ después en una localización diferente $l_{2}$, en un tiempo $t_{2}$. La primera dificultad es que no se puede representar adecuadamente esta situación, repitiendo en $t_{2}$ la misma generalización existencial que ocupamos en $t_{1}$. Supongan que el objeto $O_{1}$ es una manzana en particular. Ciertamente, la repetición de "Hay una manzana" o "Existe una manzana" no ayuda en nada, ya que estas descripciones podrían haber sido usadas por observadores diferentes, mientras que el movimiento de $l_{1}$ a $l_{2}$ solo necesita de la presencia de un observador. Una sugerencia inmediata sería incluir la localización en el contenido perceptual, diciendo, por ejemplo, "Hay una manzana $a h i$ ", refiriéndose a un lugar en un tiempo determinado; y diciendo "Hay una manzana $a h i$ ", refiriéndose a un lugar diferente en otro tiempo. A mi juicio, Sainsbury $(2005,248)$ hace un análisis certero de esta posibilidad al argumentar que es insuficiente, ya que no logra dar cuenta del

4 No consideraré formas alternativas de adscribir contenidos intencionales, por ejemplo, aquellas que contienen elementos singulares no específicos como "él” o "ella" en la expresión "Viendo una lámpara tuvo la impresión de que ella era pequeña”.

5 La presentación de estas formulaciones y la discusión de los problemas que involucra cada una de ellas se basa en lo expuesto por Sainsbury (2005). 
hecho de que, pese al cambio de locación, la manzana es representada o se nos da como la misma manzana.

¿Qué nos ofrece el "russellianismo puro", versión del generalismo según la cual podemos especificar contenidos perceptuales, usando descripciones definidas que contienen exclusivamente términos generales? La aceptación del russellianismo puro implicaría asumir que, para cada objeto de la percepción, existe una proposición o contenido general capaz de dar cuenta de la singularidad de la experiencia, esto es, capaz de individuar el objeto de una experiencia de manera única o singular. Ahora bien, la posibilidad de que descripciones exclusivamente generales puedan realizar esta tarea es muy remota. Incluso aquellos filósofos que simpatizan con la interpretación generalista adoptan una estrategia más moderada, en la que contenidos existencialmente cuantificados presentan el mundo "como conteniendo un objeto de cierto tamaño y forma, en cierta dirección, a una distancia específica del sujeto que percibe" (Davies 1992, 26). La inclusión de términos que refieren a localizaciones, direcciones o relaciones con el sujeto de experiencia es, por sí misma, un síntoma explícito de lo inadecuado que es postular un generalismo puro. Si queremos sostener la tesis generalista es indispensable adoptar la estrategia que Sainsbury llama "russellianismo impuro", que usa descripciones definidas que contienen términos singulares, como los sugeridos en la cita anterior por Davies, al menos para un conjunto limitado de entidades.

La estrategia russelliana impura más usual apela a la inclusión de elementos que especifican la dirección o la locación de objetos relativos al sujeto que percibe dentro de una descripción definida. Así, los contenidos se expresan de la siguiente forma: "Existe a lo menos y a lo más un $O$ alli, y este $O$ es $F$ ", donde $O$ denota un objeto particular - como una persona o una manzana- y $F$ se refiere a un atributo o cualidad -como "es redondo" o "tiene dos metros de altura". Para evaluar esta teoría debemos preguntar lo mismo que hemos estado preguntando en todos los casos anteriores: ¿Es el russellianismo impuro capaz de entregarnos condiciones de veracidad adecuadas?

Para contestar esta pregunta es pertinente introducir un ejemplo, sugerido originalmente por Soteriou (2000), que nos permitirá clarificar lo que está en juego ${ }^{6}$. Imaginen que un sujeto $S$ usa lentes que desplazan fenoménicamente la ubicación real de los objetos en su entorno físico. La utilización de estos lentes nos permite distinguir entre la posición aparente o "fenoménica" de un objeto (la ubicación que un objeto parece tener desde la perspectiva de $S$ ) y la posición "real" del mismo objeto (la ubicación que este objeto de hecho tiene en el entorno físico de $S$ ). En el caso que nos interesa, los lentes que usa $S$ desplazan fenoménicamente hacia la derecha la ubicación real de todos los objetos en su entorno. Imaginen que el contenido de la experiencia visual de $S$ es tal, que le parece a $S$ como si hubiera un objeto rojo y redondo

6 Soteriou (2000) usa este ejemplo para mostrar que la veracidad es algo que no puede establecerse con independencia de la especificación del contenido de una experiencia. 
frente a él. Supongan que el objeto que es causalmente responsable de la experiencia de $S$, de hecho está ubicado a la izquierda de $S$. En este ejemplo, $S$ no percibe correctamente el objeto porque-como nosotros sabemos-el objeto percibido por S no es tal cual S lo representa. En concreto, el objeto no está ubicado o localizado donde parece estarlo. Este es un caso de percepción no-verídica, producto de la acción de los lentes. Imaginen ahora el siguiente escenario alternativo:

Sin embargo, sin alterar el objeto de la experiencia y sin interferir con la dependencia causal que existe entre la experiencia del sujeto y el objeto de la percepción, podríamos arreglar las cosas de modo que la experiencia del sujeto fuera verídica. Podríamos ubicar un objeto rojo y redondo frente al sujeto, que correspondiera exactamente con el aspecto del contenido de la experiencia causado por el objeto de experiencia. La introducción de un nuevo objeto en la escena la modifica y hace que para el sujeto pareciera como si hubiera otro objeto rojo y redondo a su derecha. Este aspecto del contenido de la experiencia del sujeto puede satisfacerse mediante la introducción de un nuevo objeto rojo y redondo a la derecha del sujeto - un objeto que está demasiado a la derecha como para ser percibido por el sujeto. Noten que al sujeto no le parece como si no existiera un objeto a su izquierda y que, a pesar de que el sujeto estará percibiendo incorrectamente dos objetos rojos y redondos, el contenido de su experiencia es completamente verídico (Soteriou 2000, 180).

La posibilidad de tener una experiencia perceptual verídica claramente incorrecta es un desafío para el generalismo. Admitamos que Soteriou nos habla de objetos de la misma forma y tamaño. Llamemos al primer objeto rojo y redondo ubicado a la izquierda del sujeto que percibe "objeto ${ }_{1}$ ", al segundo objeto rojo y redondo ubicado frente al sujeto "objeto 2 ", y finalmente al objeto rojo y redondo ubicado a la derecha del sujeto "objeto ${ }_{3}$ ". Debido a que el sujeto está ocupando lentes que desplazan fenoménicamente hacia la derecha la localización real de las cosas, Soteriou está en lo cierto cuando señala que el objeto ${ }_{3}$ puede estar demasiado a la derecha y, por lo tanto, ser invisible para el sujeto. Siguiendo el ejemplo y auxiliados por las manillas de un reloj, al sujeto le parece como si hubiese un objeto rojo y redondo ubicado a la una en punto. También le parece como si hubiese otro objeto rojo y redondo ubicado cerca de las tres en punto. Nosotros sabemos que el objeto ${ }_{1}$ es el responsable causal de la aparente locación de un objeto rojo y redondo a la una en punto, y sabemos que el objeto $_{2}$ es la causa de la aparente locación de un objeto rojo y redondo a la tres en punto.

De acuerdo con el russellianismo impuro, el contenido de la experiencia de este sujeto que percibe podría ser expresado por "Hay un objeto rojo y redondo a las tres en punto". El contenido de esta percepción es verídico, pero desafortunadamente el sujeto no ve el objeto particular que de hecho es responsable de que el contenido intencional de su experiencia sea verídico, que en este caso es el objeto ${ }_{3}$. Según lo expuesto, al menos la versión del russellianismo impuro que Sainsbury (2005) discute, no nos entrega las condiciones de veracidad adecuadas que buscamos. El contenido perceptual no es verídico o verdadero en virtud del objeto particular que es causalmente responsable de la experiencia que tiene el sujeto. 
Una manera adecuada de dar cuenta de la particularidad de la experiencia sería aceptar que si desde la perspectiva del sujeto, el objeto ${ }_{2}$ parece ser un objeto rojo y redondo ubicado a las tres de la tarde, entonces el contenido de la experiencia es verdadero respecto del objeto $_{2}$ si y solo si el objeto 2 es realmente un objeto rojo y redondo ubicado a las tres de la tarde en relación al sujeto. Lamentablemente el ejemplo expuesto no captura esta intuición y el contenido es verídico, a pesar de que el sujeto percibe incorrectamente la localización de los objetos que de hecho son responsables causales del contenido de la experiencia.

La posibilidad de que una experiencia perceptual sea verídica y, al mismo tiempo, incorrecta, es un desafío concreto a la plausibilidad del russellianismo impuro, ya que nos fuerza a rechazar algo que comúnmente aceptamos como un supuesto básico sobre la naturaleza de la percepción visual. Me refiero a que lo habitual es asumir que nuestro entorno es realmente diferente a como nosotros lo representamos, porque necesariamente fallan algunas condiciones de veracidad o corrección de los contenidos intencionales de nuestras experiencias. De hecho, nuestra noción habitual de "percepción", de una u otra forma parece requerir una conexión intrínseca entre el percibir incorrectamente y la ausencia de veracidad. Por este motivo, Soteriou asegura que si queremos mantener en pie nuestro supuesto o creencia común de que en todos los casos de percepción incorrecta, el contenido perceptual no debe ser verídico, entonces "no deberíamos permitir que la cuestión de la veracidad (veridicality) de una experiencia se establezca independientemente de si hay o no un objeto siendo percibido" $(2000,180)$.

Según los ejemplos provistos por Soteriou, es evidente que la posibilidad de contenidos verídicos e incorrectos es un reto problemático para todas las versiones del generalismo que hemos estado discutiendo. No pretendo haber establecido de manera concluyente que el generalismo es falso. De hecho, existen otras versiones de la tesis generalista que no he incluido en mi análisis crítico, que eventualmente podrían posicionarse como alternativas interesantes ${ }^{7}$. Sin embargo, es importante enfatizar que todas las alternativas que conozco tienden a incluir lo que Sainsbury (2005) llama "objetos especiales" como parte de los contenidos perceptuales, objetos que en último término intentan captar la singularidad de la experiencia de una manera extremadamente sofisticada, que no refleja la manera en que nosotros percibimos objetos y propiedades mundanas a nivel fenomenológico y consciente. Si es que existen contenidos cuyos referentes son objetos particulares, ¿por qué no permitimos que cualquier

7 Me refiero específicamente a la postura de Searle (1983) en la que los contenidos intencionales de la percepción incluyen aspectos causales autorreferentes. Según esta teoría, las relaciones causales no son algo "extra", sino que son intrínsecas a los contenidos representacionales de la experiencia. Si bien en principio esta teoría podría entregarnos condiciones de veracidad adecuada, existen otros motivos familiares de naturaleza fenomenológica y causal que atentan contra su plausibilidad y que han sido extensamente considerados en la literatura filosófica especializada. Para una discusión en profundidad de estos aspectos, sugiero ver las críticas que se incluyen en LePore \& Van Gulick (1991). 
objeto particular sea el referente, en vez de limitar la gama de posibilidades a "objetos especiales" dentro de una estrategia general? ${ }^{8}$

Más allá de introducir construcciones complejas en la especificación de los contenidos perceptuales para dar cuenta de la naturaleza única o singular de nuestra experiencia, parece más sensato y económico especificar el contenido de una percepción, incluyendo elementos demostrativos que refieran directamente a los objetos particulares en cuestión. Esta es sin duda la intuición que justifica la búsqueda de una alternativa singularista para la especificación de contenidos perceptuales. Así, los contenidos de nuestras percepciones de objetos mundanos -como esa mesa o esta manzana roja y redonda- estarán constituidos, es decir, vinculados ontológicamente con los objetos singulares que percibimos ${ }^{9}$.

\section{Percepción, alucinación y conceptos demostrativos}

Las dificultades inherentes a la tesis generalista justifican la búsqueda de una alternativa singularista. Ahora bien, debemos tener claro que la aceptación de una postura singularista está directamente relacionada con la adopción de un rol esencial de los objetos de la percepción, no solo en el proceso de individuación de los contenidos, sino también determinando el tipo de experiencia que tenemos. Ya no se trata de una postura en la cual los objetos de nuestro entorno meramente "calzan" o "corresponden" con los especificado en los contenidos intencionales, sino que ahora los objetos mismos "entran", "constituyen" o "son parte" de los contenidos. Esta estrategia intenta realzar la naturaleza relacional de los estados perceptuales y, al mismo tiempo, dejar en claro que la percepción y la veracidad deben ir intrínsecamente de la mano.

8 Dentro de estos objetos especiales habría que estipular, por ejemplo, aquellos objetos supuestos por las estrategias que intentan incluir al sujeto que percibe en el contenido, como en "Existe solo un objeto-visto-por-mí a las tres de la tarde que es rojo y redondo", o simplemente aspectos casuales auto-referenciales como lo intenta Searle (1983). Concuerdo con Sainsbury (2005), en que los intentos por incluir objetos especiales como aquellos "objetos-vistos-por-mi" son síntomas de la falla del generalismo cuando intenta dar cuenta de manera directa de la estructura relacional de nuestra percepción.

9 Un análisis o definición rigurosa de "constitución" es una compleja tarea de la metafísica que sobrepasa las pretensiones del presente artículo. Sin embargo, hay ciertos elementos que parecen aplicarse al caso de las experiencias visuales y que podrían servirnos para comprender cuando es una relación de constitución (espacial o temporal) lo que está en juego, y no un tipo diferente de relación. Concuerdo con Benj Hellie (manuscrito no publicado) en que estos elementos pueden ser captados por las ideas de (a) co-presencia de $x$ e $y$, y (ii) dependencia existencial asimétrica de $x$ en $y$. Hellie no asegura que si (i) y (ii) son satisfechos por un par cualquiera, entonces necesariamente se trata de un caso de constitución. Más bien, su punto es que (i) y (ii) "sugieren fuertemente que un miembro del par constituye al otro y que, de hecho, que esto sea así es una atractiva explicación prima facie de por qué (i) y (ii) se satisfacen" (manuscrito no publicado, 40). Para una discusión en profundidad sobre la relación de constitución invito al lector a revisar Lowe (2002), Child (1994, 161) y Johnston (1992). 
Paul Snowdon (1981) fue quizás uno de los primeros filósofos en argumentar enfáticamente que nuestra noción ordinaria de percepción -particularmente nuestra idea de una conciencia visual directa de objetos en nuestro entorno físico- está vinculada esencialmente con la posibilidad que tenemos de emitir juicios demostrativos verdaderos de la forma "Eso es $F$ ". Si los conceptos demostrativos son aquellos conceptos "cuya comprensión depende esencialmente de la relación de un sujeto con las entidades reales que constituyen su valor semántico" (Brewer 1999, 171), entonces la caracterización de contenidos visuales mediante el uso de demostrativos desempeña un rol fundamental al distinguir entre instancias propiamente perceptuales e instancias meramente alucinatorias.

La inclusión de demostrativos perceptuales en el contenido de una experiencia nos permite restablecer el supuesto de que los contenidos de la percepción están siempre relacionados con los objetos causalmente responsables de la experiencia. El que una experiencia incluya demostrativos perceptuales singulares como parte del contenido intencional nos permite descartar inmediatamente escenarios no-perceptuales. Por ejemplo, podemos descartar aquellas instancias en las que los demostrativos fallan a nivel referencial como situaciones en las que los contenidos no son verídicos. Así, en oposición a la interpretación del generalismo, esta estrategia singularista asegura que la veracidad de un contenido no sea algo ajeno al acto de percepción.

Ahora bien, asumiendo que la naturaleza relacional de los contenidos visuales que involucran esencialmente demostrativos como "Ese es $F$ " es clave para comprender la percepción, es importante pensar acerca del rol que juegan estos elementos singulares demostrativos durante experiencias alucinatorias indistinguibles de la percepción. Una estrategia popular para entender la función de los demostrativos en este contexto es la que está implícita en las interpretaciones singularistas de Evans (1982) y McDowell (1982, 1998a, 1998b). En su clásico Variedades de la Referencia, Evans introduce pensamientos russellianos, es decir, pensamientos compuestos por sus objetos referenciales (object-involving thoughts). Evans dice que "un pensamiento es russelliano si es que es de una clase que simplemente no podría existir en la ausencia del objeto o los objetos de los que ese pensamiento trata" $(1982,71)$ y McDowell (1998a), al discutir la posibilidad de contenidos de re, argumenta que cualquier visión en la que un objeto pueda ser pensado de manera demostrativa, aunque este no exista realmente, es algo inaceptable y difícil de tolerar. La conexión entre estas teorías de contenidos russellianos y la posibilidad de alucinaciones que los sujetos no son capaces de discriminar de la percepción, nos conduce a una forma de externismo de contenidos expresada adecuadamente en un pasaje de Evans:

Por lo tanto, los pensamientos demostrativos sobre objetos, como pensamientos- 'aquí' son russellianos. Si no hay un objeto con el cual el sujeto está de hecho en 'contacto' informacional -si él está alucinando, o si muchos objetos diversos se suceden sin que él lo note- entonces él no tiene una idea-de-unobjeto-particular, y por lo tanto, no tiene pensamiento. Su pensamiento demostrativo acerca de un objeto particular depende del hecho de una conexión informacional de clase, no del pensamiento o idea de esa conexión; y, por tanto, imposible de interpretar, si es que no hay objeto con el cual él esté conectado (Evans 1982, 173). 
Evans y McDowell parecen estar de acuerdo en que tanto la existencia de contenidos, como la existencia de los episodios de pensamiento/experiencia dependen esencialmente de la existencia real de los objetos representados ${ }^{10}$. En relación con las alucinaciones, la interpretación que ellos proponen conlleva una radicalidad que es difícil de aceptar. Me refiero al hecho de que, a juicio de Evans y McDowell, las alucinaciones no solo no tienen contenidos, sino que no pueden ser consideradas en lo absoluto experiencias genuinas. Para ilustrar adecuadamente la radicalidad de esta propuesta, los invito a reflexionar sobre la base de la siguiente situación. Imaginen que ustedes participan en un experimento psicológico donde se encuentran sentados, en una habitación completamente blanca, con cables conectados a su cabeza. Estos cables se comunican con un computador capaz de enviar impulsos eléctricos que estimulan su corteza cerebral, induciéndoles alucinaciones que ustedes no pueden discriminar introspectivamente de instancias normales de percepción. El investigador a cargo del experimento puede conectar o desconectar el dispositivo encargado de enviar los impulsos sin que ustedes noten diferencia alguna. Asumiendo la posibilidad de esta situación, consideren los siguientes escenarios, donde el sujeto $\mathrm{S}$ podría ser cualquiera de ustedes:

(a) El investigador conecta los cables a la corteza cerebral de S. Sobre la base de como son visualmente las cosas desde su perspectiva, S no duda en juzgar "Eso es rojo". De hecho, al frente suyo hay una manzana roja. El dispositivo estuvo en todo momento apagado. Por lo tanto, $S$ realmente vio una manzana roja.

(b) Después de un momento, el psicólogo investigador le pregunta nuevamente a $S$ cómo son las cosas visualmente para él. Sobre la base de la información disponible a nivel introspectivo, S inmediatamente vuelve a juzgar "Eso es rojo". En este caso el dispositivo estaba activado y no había ninguna cosa roja frente al sujeto. $S$ estaba solo alucinando.

McDowell y Evans afirmarían que (a) es claramente una instancia de percepción en la que un objeto particular es parte del contenido de la experiencia y necesariamente debe figurar en su especificación. Sin embargo, la teoría objetual ${ }^{11}$ de contenidos singulares que ellos ofrecen implica que durante situaciones del tipo (b), donde no hay objetos que puedan ser percibidos, los sujetos simplemente no tienen experiencias con contenido. Esto es prometedor en cuanto ofrece una visión en la que hay una dependencia asimétrica fundamental entre situaciones perceptuales y noperceptuales. Pero también implica consecuencias que a mi juicio son muy fuertes y difíciles de aceptar. pensamientos, sin embargo ellos "figuran en" los pensamiento como sentidos de re (McDowell 1998a, 237).

11 Utilizaré los términos "teoría objetual" o "contenido objetual" para referirme a la teoría filosófica según la cual los contenidos perceptuales dependen esencialmente de los objetos particulares que representan. 
Establecer una dependencia de los contenidos singulares respecto de sus objetos equivale a afirmar que los contenidos no estarían disponibles para ser pensados o experimentados en la ausencia de dichos objetos. Esto es alentador, pero según McDowell y Evans, todo episodio de pensamiento o experiencia está, al mismo tiempo, constituido por la disponibilidad de contenidos. Por lo tanto, la teoría que ellos defienden tiene como consecuencia no solo el hecho de que un sujeto que alucina no puede saber si hay o no un objeto que es percibido, sino además el hecho de que el mismo sujeto puede estar ofuscado respecto de si realmente se encuentra pensando o teniendo una experiencia. En otras palabras, para McDowell y Evans las alucinaciones no son experiencias, sino solo "ficciones" o "simulaciones" de episodios mentales ${ }^{12}$.

En mi opinión, sugerir que las alucinaciones no son experiencias genuinas - tal como McDowell y Evans lo hacen- es un error. Es importante recordar que no podemos discriminar las alucinaciones de sus contrapartes perceptivas desde el punto de vista introspectivo. Para cada alucinación podría existir una percepción fenomenológicamente indiscriminable. De hecho, el breve experimento mental que presentamos anteriormente ilustra que los sujetos tienden a realizar el mismo juicio en escenarios del tipo (a) y (b), porque no pueden diferenciar introspectivamente (a) de (b). Si los sujetos pueden hacer el mismo tipo de juicios sobre la base de cómo las cosas parecen ser en (a) y (b), entonces ciertamente pueden formar creencias que, en conjunción con deseos asociados, producen acciones o comportamientos similares. Si esto es así, entonces las alucinaciones y las percepciones pueden desempeñar roles psicológicos estrechos (narrow) similares, a pesar de sus diferentes naturalezas constitutivas.

El hecho de que las alucinaciones sean fenomenológicamente indiscriminables de las percepciones y puedan jugar roles psicológicos similares en conexión con la formación de creencias y la activación de comportamientos son, pienso yo, buenas razones, no solo para considerarlas experiencias genuinas, sino también como experiencias que poseen contenidos singulares (aunque de constitución diferente y fundamentalmente incorrectos) que pueden especificarse utilizando expresiones del tipo "Ese $F$ es $G$ ". Eso sí, es importante enfatizar que la naturaleza objetual (objectinvolving) de los contenidos singulares tiene como consecuencia directa el que los contenidos de las experiencias perceptuales (percepciones) y sus contrapartes noperceptuales (alucinaciones) sean de un tipo fundamentalmente diferente. No me refiero simplemente a que, a diferencia de los contenidos perceptuales, los contenidos alucinatorios son falsos o incorrectos. Más bien, lo que está en juego es una idea fuerte, según la que "los contenidos singulares no-vacíos son esencialmente no-vacíos, y los contenidos singulares vacíos son esencialmente vacíos, así no existe contenido

12 Las "ficciones" o "simulaciones" de experiencia se diferencian de experiencias genuinas de la misma forma en que una manzana falsa o de mentira (de porcelana, por ejemplo) se diferencia de una manzana real. Es en este sentido que según McDowell y Evans estos "mock thoughts or experiences" se diferencian de las experiencias reales. 
singular alguno que sea común a la percepción y la alucinación" (Sainsbury 2005, $73)^{13}$.

La estrategia que he sugerido no solo garantiza una demarcación ontológica fundamental entre casos perceptuales "buenos" y casos "malos" de alucinación. Al mismo tiempo, provee un sistema filosófico que es totalmente compatible con aspectos teleológicos relacionados con nuestra capacidad de representar correcta e incorrectamente nuestro entorno físico. Los contenidos de nuestras experiencias visuales tienen la función o propósito natural de entregarnos información sobre el ambiente físico. Sin embargo, en ocasiones, los contenidos singulares de algunas experiencias no se comportan en concordancia con su función natural y fallan a nivel referencial. La estrategia propuesta -a diferencia de la interpretación propuesta por McDowell y Evans- se hace cargo de esta posibilidad de error, sin negar el carácter experiencial e intencional de este tipo de episodios mentales. Las alucinaciones, pese a no producir representaciones adecuadas de nuestro entorno físico y fallar a nivel referencial, conservan su estatuto de experiencias genuinas con contenido singular ${ }^{14}$.

\section{Elementos singulares primitivos}

Existe un asunto específico que podría preocupar a algunos filósofos que adoptan la postura recién expuesta. Me refiero a que usualmente se piensa que solo podemos establecer la singularidad de los contenidos visuales si aceptamos que estos son esencialmente proposicionales y, por lo tanto, en principio evaluables en términos de verdad o falsedad. El que los contenidos sean esencialmente proposicionales significa que ellos están siempre totalmente estructurados y compuestos por conceptos que los sujetos deben poseer e implementar durante la experiencia. Así, la supuesta singularidad de los contenidos sería una consecuencia directa del hecho de que nuestras percepciones se estructuran fundamentalmente del mismo modo en que se estructuran actitudes proposicionales como la creencia o el deseo. Si esto es así, la falla de los contenidos visuales a nivel de referencia demostrativa siempre tendrá como consecuencia la requerida distinción entre percepciones y alucinaciones que hemos establecido.

13 Esta interpretación es compatible con la visión filosófica que asegura que las experiencias alucinatorias con contenido son parasíticas en percepciones con contenido. Así, las experiencias visuales "malas", cuyos contenidos no tienen un objeto o propiedad como referente son "asimétricamente dependientes" de aquellas experiencias que refieren exitosamente a los objetos/ propiedades que representan. Adhiero plenamente a esta primacía ontológico-teleológica de la percepción por sobre la alucinación.

14 Las alucinaciones son experiencias visuales con contenido singular. Ahora bien, son experiencias que no llevan a cabo adecuadamente su función o telos natural. Esto es análogo a la forma en que, por ejemplo, un hígado malformado sigue siendo un hígado, pese a que no desempeña adecuadamente su función natural de filtrar en nuestro organismo. 
La preocupación es la siguiente: ¿Qué ocurriría si las proposiciones (evaluables en términos de verdad y falsedad) que ocupamos para especificar o caracterizar estos contenidos no son realmente esenciales para estos contenidos? Uno es capaz de describir el contenido de una experiencia perceptual de un objeto en particular diciendo algo como "Me parece que aquel $F$ es $G$ ". Podemos asumir que los contenidos visuales son describibles en términos proposicionales, pero esto no nos fuerza a aceptar que estos contenidos son proposicionales, de la forma en que sí los son los contenidos de las creencias. Tampoco nos fuerza a asegurar que estos contenidos no pueden ser atribuidos a criaturas -infantes y animales- que no poseen las habilidades conceptuales y lingüísticas necesarias (incluyendo demostrativos perceptuales) para la especificación canónica de estos contenidos.

Si los contenidos de la percepción no son esencialmente proposicionales de la forma en que sí lo son los contenidos de las creencias, entonces uno podría razonablemente cuestionar algunas de las teorías que hemos expuesto y preguntarse si es que hay recursos adicionales para respaldar la tesis de que los contenidos de la percepción contienen elementos singulares ${ }^{15}$. Consideren, por ejemplo, la siguiente nota al pie que Mark Sainsbury incluye en su argumento contra la tesis "generalista":

En esta parte de la discusión, no es fácil mantener mi neutralidad oficial sobre la cuestión de si el contenido perceptual es conceptual o no-conceptual. Yo asumo que el contenido no-conceptual no es inefable: al menos parte de él puede ser especificado lingüísticamente, tal como lo requieren las versiones del generalismo que hemos discutido aquí... Podría ser el caso de que una versión más robusta de contenido no-conceptual, de acuerdo a la cual se trata de un tipo de contenido distintivo y no sólo de una forma distintiva de poseer un contenido, no haga sentido de las distinciones que esta discusión requiere (por ejemplo, entre singular y general). Esto sería tan antitético a las teorías que me opongo, como a aquellas que estoy defendiendo (Sainsbury 2005, 247).

Pese a que aquí no se discutirá en detalle en torno a la distinción entre contenidos de naturaleza "proposicional conceptual" y aquellos contenidos "no-conceptuales", pienso que la preocupación de Sainsbury es genuina y que es necesario decir más acerca de este asunto. Por lo menos, me parece necesario suplementar la inquietud de Sainsbury con recursos que nos permitan mantener sus intuiciones respecto de la singularidad de los contenidos de la percepción, incluso a un nivel representacional nolingüístico o no-conceptual. Para ello introduciré algunas consideraciones provenientes de investigaciones empíricas en las ciencias cognitivas. En particular, me parece relevante resaltar investigaciones recientes que sugieren la existencia de elementos singulares de la experiencia visual que rastrean objetos particulares en nuestro entorno y que operan de una manera análoga a los conceptos demostrativos perceptuales.

15 En otras palabras, si pensamos que los contenidos perceptuales no son esencialmente proposicionales y evaluables en términos de verdad o falsedad, entonces la supuesta singularidad de la experiencia visual podría no establecer la buscada distinción fundamental entre percepciones y alucinaciones. 
La investigación empírica de Zenon Pylyshyn (2003) establece la existencia de lo que este destacado científico llama "F.I.N.S.T" (Fingers of Instantiation Index Mechanism) o simplemente "índices" o "indexicales visuales". El objetivo de Pylyshyn es establecer una correspondencia o vínculo adecuado entre las representaciones visuales y el mundo. De un modo similar al que hemos explorado de manera filosófica, Pylyshyn piensa que existe una diferencia fundamental entre representar el hecho general de que existe algo que posee ciertas propiedades y representar el hecho de que esta cosa particular (singular) posee aquellas propiedades. Esto es por supuesto otra manera de realizar el contraste entre modelos de representación mental puramente descriptivos (generales) y modelos que involucran referencia directa a objetos particulares en el mundo (singulares). De acuerdo a Pylyshyn, el modelo descriptivo -similar al que nosotros hemos llamado generalismo- es insatisfactorio cuando intenta explicar algunas propiedades de nuestro sistema visual y dar cuenta de la conexión evidente que existe entre la visión y la acción. Para explicar estos fenómenos, se requiere que las representaciones visuales incluyan necesariamente elementos similares a los demostrativos perceptuales que funcionan a un nivel causal más primitivo, sin la mediación de descripciones, y con anterioridad a cualquier proceso de codificación conceptual.

Lo que necesitamos es lo que el lenguaje natural provee en parte, cuando utiliza nombres (o etiquetas) que denotan únicamente individuos particulares o cuando incluye demostrativos, términos como "esto" o "aquel". Con este tipo de mecanismos podemos referir a individuos particulares y, al hacerlo, podemos elaborar descripciones, mientras tenemos la garantía de que continuamos describiendo la misma cosa. Además, también rastrear un objeto particular e individual como el mismo objeto, incluso si este cambia sus propiedades y se mueve de forma impredecible. Este último requerimiento debería dejar claro que la función que necesitamos es precisamente lo propuesto en la teoría de los índices visuales. De acuerdo a esta teoría, los índices visuales ejecutan una labor equivalente a la referencia directa, similar a un demostrativo, dentro del sistema visual (Pylyshyn 2003, 254-255).

El proyecto de Pylyshyn no se remite a decir que necesitamos índices visuales para rastrear objetos particulares en el entorno. Su investigación incluye evidencia empírica que ilustra que nuestro sistema visual incluye mecanismos que realizan labores semejantes a aquellas realizadas por expresiones demostrativas y deícticas en el lenguaje natural. Dentro de la evidencia considerada hay diversos experimentos sobre atención visual. Uno de ellos es el multiple-object-tracking task (MOT), donde los observadores se exponen a una pantalla que contiene ocho objetos simples e idénticos que se mueven de forma impredecible. Al comienzo del experimento, un subconjunto de estos objetos se colorea de un modo diferente. El objetivo del observador es rastrear (to keep track of) estos objetos que se mueven aleatoriamente.

Al cabo de varios experimentos que comprendían 4 objetos coloreados, el desempeño humano llegó a ser correcto en aproximadamente un $88 \%$ de los casos. Pylyshyn argumenta que una de las explicaciones posibles de este fenómeno es que los observadores "registran y usan las locaciones de cada uno de los objetos y las visitan de 
manera serial" (2003, 224). Sin embargo, la velocidad de las pruebas de escaneo atencional sugieren que esta estrategia no podría tener un desempeño correcto superior al $10 \%$ cuando realmente se rastrean los objetivos o superior a un $40 \%$ si es que incluimos la posibilidad de adivinar. De hecho, un resultado común en estos casos seriales es que los sujetos terminan rastreando objetos equivocados. ¿Cómo es posible entonces explicar un asombroso desempeño humano de $88 \%$ ?

...lo observadores no podrían haber mantenido un rastreo de los objetivos utilizando una descripción única registrada para cada objeto, ya que en cada instante de tiempo la única propiedad propia de cada objeto es su localización. Si yo tengo la razón al argumentar sobre la base de la naturaleza de los parámetros de rastreo, que las localizaciones registradas no se pueden utilizar como base de los seguimientos, entonces todo lo que nos queda es la individualidad del objeto (Pylyshyn 2003, 226).

Los resultados del experimento, en conjunto con los parámetros de rastreo o seguimiento mencionados, sugieren que debe existir algo así como un índice visual capaz de rastrear y mantener la identidad de los objetos. Según Pylyshyn, los mismos resultados se desprenden de experimentos basados en la selección de objetos durante los que los psicólogos y científicos cognitivos denominan "search tasks". Estos seguimientos o search tasks son situaciones en las que observadores deben identificar objetos únicos, pese a la existencia de elementos distractivos. Además, durante estos seguimientos, los observadores deben hacerse cargo de dos fenómenos visuales. El primero se conoce como "inhibición del retorno", y se produce cuando nuestra atención demora más tiempo de lo habitual en volver a un objeto particular que pudo haber cambiado su locación. El segundo se conoce como el fenómeno visual de "subitización", donde los objetos son individuados rápidamente y en forma preatencional.

La lección empírica de estos seguimientos es que "el sistema motor no puede individualizar objetos en virtud de una descripción única; este tiene que tener alguna forma más directa de referencia a ellos" (Pylyshyn 2003, 244). Lo que se requiere es un mecanismo capaz de identificar objetos distantes individuales en cuanto individuos y no meramente por el hecho de satisfacer una descripción general registrada en el cerebro después de episodios perceptuales anteriores o propiedades generales que el objeto podría satisfacer, como, por ejemplo, la locación. La evidencia empírica recopilada por Pylyshyn y otros psicólogos cognitivos sugiere la existencia de un elemento de nuestro sistema de representación visual que juega un rol similar al jugado por los términos indéxicos en la filosofía del lenguaje. Se trata de la existencia de un índice visual capaz de rastrear e identificar objetos visuales de manera individual y sin perder su identidad. De hecho, parece ser que solo podemos determinar propiedades generales de los objetos (que podrían ser parte de una descripción general) una vez que este vínculo referencial directo con los objetos ya se ha establecido. 


\section{Comentario Final}

Iniciamos esta discusión destacando el hecho de que una de las visiones filosóficas más difundidas dentro del debate contemporáneo acerca de la naturaleza de la percepción visual adopta una postura de corte intencional e internista. "Intencional", porque explica nuestra capacidad de ver cosas (objetos, propiedades) localizadas en el entorno en términos de nuestra capacidad para tener experiencias visuales con contenidos intencionales, e "internista", porque supuestamente las cosas localizadas en nuestro entorno físico - tal como lo sugieren las alucinaciones verídicas-no constituyen esencialmente los contenidos intencionales de nuestra experiencia. Una de las consecuencias directas de este enfoque internista es la supuesta naturaleza "general" de los contenidos de la percepción. Según el generalismo, los contenidos visuales nunca incluyen esencialmente los objetos particulares que percibimos y, por lo mismo, no deben especificarse usando términos singulares.

En este artículo he argumentado -sobre la base de consideraciones filosóficas (Sainsbury 2005; Soteriou 2000) y empíricas (Pylyshyn 2003, 2000)- que el generalismo no es el enfoque adecuado para comprender la naturaleza de los contenidos perceptuales. En particular, la tesis generalista no es capaz de dar cuenta de que la veracidad de nuestras experiencias perceptuales no es un asunto totalmente independiente del hecho de si percibimos o no. A mi juicio, este último elemento es un aspecto central de nuestra entendimiento ordinario de la noción de "percepción" y de lo que implica percibir objetos y propiedades en nuestro entorno.

Los problemas que enfrenta el generalismo nos llevaron a considerar seriamente una alternativa "singularista" o "particularista", según la cual los objetos mundanos efectivamente son parte de los contenidos de la percepción. Esto nos permite proponer que las percepciones y las alucinaciones son experiencias esencialmente diferentes, a pesar de que no son introspectivamente discriminables y que pueden jugar roles psicológicos estrechos similares en la formación de creencias y la dirección de nuestro comportamiento. Esta última observación es de gran importancia, ya que nos ayuda a entender que la presencia necesaria de elementos singulares, junto a la naturaleza objetual de los contenidos de la percepción, no afectan nuestra apreciación de las alucinaciones como experiencias genuinas con contenido intencional.

\section{Referencias bibliográficas}

Brewer, Bill (1999), Perception and Reason. Oxford; New York: Clarendon Press; Oxford University Press.

Child, William (1994), Causality, Interpretation, and the Mind. Oxford: Oxford University Press.

Crane, Tim (2006), "Is there a perceptual relation?", en Gendler, T. \& Hawthorne, J. (Ed.), Perceptual Experience. Oxford University Press. 
Davies, Martin (1992), "Perceptual content and local superveniente". Proceedings of the Aristotelian Society. 92: 21-45.

Evans, Gareth (1982), The Varieties of Reference. Oxford; New York: Clarendon Press; Oxford University Press.

Grice, Herbert P. (1961). "The Causal Theory of Perception". Proceedings of the Aristotelian Society, Supplementary Volume XXXV, pp. 121-153.

(1988). "The Causal Theory of Perception". En Dancy, J. (Ed.). Perceptual Knowledge. Oxford: Oxford University Press. Pp. 66-78. (Versión abreviada de Grice, 1961).

Hellie, Benj (manuscrito no publicado), Visual Phenomenal Caracter and Theories of Vision.

Johnston, Mark (1992), "Constitution is not identity". Mind. Vol. 101, 401: 89-105.

LePore, Ernest \& Van Gulick, Robert (1991), John Searle and his critics. Oxford: Basil Blackwell.

Lowe, Jonathan (2002), A Survey of Metaphysics. Oxford: Oxford University Press.

McDowell, John (1982), “Truth value gaps”, en L. J. C. et al. (Ed.), Logic, methodology and philosophy of science: Proceedings of the sixth international congress of logic, methodology and philosophy of science. New York: North-Holland Publisher Co.

(1998a), "De re senses", en Meaning, Knowledge, and Reality (pp. ix, 462 p.). Cambridge, Mass.: Harvard University Press. 403-432.

(1998b), "Replies". Philosophy and Phenomenological Research. 58:

McGinn, Colin (1982), The Character of Mind. Oxford; New York: Oxford University Press.

Pereira, Francisco (2006), "Metaphysical Disjunctivism and the Intentional Theory of Perception", en Di Nucci, E. \& McHugh, C. (Eds.), Content, Consciousness, and Perception: Essays in Contemporary Philosophy of Mind. London: Cambridge Scholars Press.

Pylyshyn, Zenon W. (2000), "Situating vision in the World", Trends in Cognitive Science, 4 (5).

(2003), Seeing and Visualizing: It's not what you think. Cambridge, Mass.: MIT Press.

Sainsbury, Mark (2005), Reference without Referents. Oxford [England] New York: Clarendon Press; Oxford University Press.

Searle, John R. (1983), Intentionality. Cambridge Cambridgeshire; New York: Cambridge University Press.

Snowdon, Paul (1981), "Perception, vision, and causation", Proceedings of the Aristotelian Society. 81: 175-192.

Soteriou, Mathew (2000), “The particularity of visual perception”, European Journal of Philosophy, 8 (2), 173-189.

Tye, Michael (2000), Consciousness, Color, and Content. Cambridge, Mass.: MIT Press. 